\title{
A FRAMEWORK TO AUTOMATICALLY MONITOR THE POSITION OF SITE RESOURCES WITH LOW-ACCURACY ESTIMATES
}

\author{
David Grau* \\ Department of Civil, Construction and Environmental Engineering, \\ The University of Alabama, Tuscaloosa, USA \\ Corresponding author (dgrau@eng.ua.edu)
}

\begin{abstract}
Large productivity drawbacks have been historically related to the inefficient flow of site resources. Increments in accrued costs and time and safety incidents result from the flow or movement of resources across the job sites. Even though the importance of this problem, there is not data to characterize the unnecessary movement of site resources. This fact is a consequence of the overwhelming burden that such characterization would give rise to with traditional control techniques based on human observations and paper based records. Currently, though, large contractor organizations have started to take slow but seemingly decided steps towards a more visible control of their site resources with infrastructure-less tracking approaches that result in low-accuracy locations. Efficiently identifying the movement of site resources throughout the job site with low-accuracy location estimates results in a non-trivial problem that cannot be properly solved using deterministic or classic probabilistic approaches. Instead, the author proposes an innovative approach based on the adoption of belief reasoning functions. In this approach, a resource is believed to actually be in a new set coordinates if and only if $t$ he sum of conflicting values as a result of intersecting pairs of confidence circles centered in the last and predecessor coordinated estimates exceeds a pre-defined threshold of uncertainty. The quantitative approach is validated against empirical observations. The results indicate that the proposed method efficiently detects the actual movement of resources on the job site and correctly filters location uncertainties.
\end{abstract}

Keywords: Materials, Labor, Tracking, Real-time, Automation, Monitoring, Productivity.

\section{MOTIVATION}

Large productivity drawbacks have been historically related to the inefficient flow of site resources. Increments in accrued costs, and time and safety incidents result from the flow or movement of resources across the job sites. When the movement of a resource is unnecessary from a production perspective, the consumed time and costs are left uncompensated. Double-handling of materials is a common example of the unnecessary movement of site resources. Thus, significant amounts of expenditures are accrued when most material components are displaced to new coordinates, since their movement requires the utilization of expensive lifting and hauling equipment in addition to craft workers hours. Therefore, if the movement of a material is unnecessary, one can also argue that an opportunity for effectively utilizing the work-time of equipment units and workers is being missed. Ideally, the displacement of site resources (i.e. materials, equipment, and labor) should be adjusted to simply satisfy construction requirements and hence to maximize craft labor and equipment productivity ratios.

The previous reasoning is consistent with lean production thinking. Lean production, a manufacturing approach originally conceptualized by Toyota during the early seventies, is sustained by the reduction of waste, by improved planning and control mechanisms, and by the efficient orchestration of available resources. Thus, lean thinking implicitly emphasizes the need for a smooth flow of resources in order for a production process to be effective. Thus, the timely monitoring of site resources is a sine qua non 
requirement for efficient construction operations. Hence, under the lean prism, the unnecessary movement of site resources is also a clear form of waste that needs to be eradicated.

Currently, large contractor organizations have started to take slow but decided steps towards a more visible control of their site resources with infrastructure-less tracking approaches [1]. While these tracking technologies can result in positive benefit to cost ratios, they also frequently result in low-accuracy localization estimates. Thus, it is difficult to assess whether or not a resource has moved/been moved to a different position based on the location estimates alone. While other technologies exist that can actually provide a precise localization of construction resources, these technologies can only operate at the expense of a dense deployment of infrastructure assets and large upfront and maintenance costs.

On top of this low positioning accuracy, construction job sites pose implicit challenges associated with their time varying, harsh, and unpredictable conditions. Indeed, these conditions radically change the behaviour of the signals required for the operation of tracking technologies, further decreasing their localization accuracy. While low localization accuracies have been demonstrated to be sufficient for reliably locating a site resource [1, 2, 3], they can render totally impossible for the end user to reliably know when different estimated coordinates for a given resource are the result of either the sum of multiple sources of inaccuracy or the actual movement of the resource to a new pair of coordinates. This problem is aggravated by the fact that some resources, such as labour workers engaged in a given activity, frequently travel in very short distances that prevent tracking the worker with low accuracy technologies without the appropriate reasoning approaches.

Thus, monitoring the movement of site resources with low-accuracy estimates results in a non-trivial problem that cannot be addressed with deterministic or classic probability approaches. Instead, this study proposes a framework based on belief functions in order to address this fundamental problem, the solution of which promises to improve the stagnant productivity of the construction industry and characterize the movement of site resources with factual data.

The rest of this paper is organized as follows. A summary of the state of the art on belief functions as a generalization of the Bayesian probability theory is presented in the next section. Then, a belief framework to monitor the movement of site resources with cost-efficient sensing approaches is introduced. This framework is validated against empirical observations. Finally, the conclusions summarize the findings of this research to the present date and introduce the necessary steps to complete this study.

\section{BACKGROUND}

The background section briefly introduces the reader to both the belief and the Bayesian probability theories. It also discusses a completed research effort in the topic of discussion. The background section follows in the paragraphs below.

\subsection{The Belief Theory}

Dempster [4] and Shafer [5] separately introduced what has been later known as the belief (and plausibility) theory. This generalization of the Bayesian body of knowledge has been successfully applied in multiple fields of investigation and practice, such as economics, engineering, and defense, among many others $[4,5,6]$. In his own words, Schafer quoted that the dissimilarity between the belief reasoning and the Bayesian probability lies in that "whereas the Bayesian theory requires probabilities for each question of interest, belief functions allow us to base degrees of belief for one question on probabilities for a related question" [7]. Thus, probability distributions may or may not exert and influence on these degrees of belief. This is equivalent to say that the degrees of belief can be based on subjective judgment. Hence, the fact of betting in the outcome of a dice is solely based on individual belief given that the each of the dice faces has the same 
probability. Because there is no possible reasoning or prior facts that can help the individual to assess the outcome of the bet, the bet is solely based on the individual's belief.

In belief theory, for each belief function $\theta$, a mass number of probability is defined for each subset $A$ of $\theta$ and noted as $\mathrm{m}(A)$. For the purpose of this study, the reader can associate a belief function with an event and its equivalent mass functions with the possible outcomes of the event based on external observations. Individually, the mass function for each subset A ranges between $[0,1]$. Since the outcome of a given event needs to actually be included in all the possible subsets, the addition of the mass functions for the distinct subsets must include all the possible ouctomes. This is mathematically translated into an axiom that requires the sum of the mass function values for all the subsets $A$ of a given event to be equal to 1 and becomes a fundamental differentiator from the traditional or classic probability. In traditional probability theory, probabilities can only be assigned to individual points in the spectrum of outcomes contained in $\theta$.

When more than one observation simultaneously exists for the same event, they need to be properly combined. For this purpose and in front of more than one item of evidence, Dempster defined the joint mass as the product of the individual mass functions in agreement divided by the product of individual mass functions resulting in a non-null intersection. This combination rule is shown in Eq. 1 for two different bodies of evidence with their corresponding mass functions $\mathrm{m}_{1}$ and $\mathrm{m}_{2}$. The empty intersection $(\psi)$ between the mass functions indicates the conflict between the coexisting observations $m_{1}$ and $m_{2}$ and is expressed by the second member in the denominator. This level of conflict reflects the degree of disagreement between two different observations, a degree of disagreement that ranges between $[0,1]$. A conflict value of 1 implies that the two observations are non-intersecting and hence are in disagreement.

$$
m(A)=\frac{\sum\left\{m_{1}(B) \times m_{2}(C) \mid B \cap C=A\right\}}{1-\sum\left\{m_{1}(B) \times m_{2}(C) \mid B \cap C=\psi\right.}
$$

\subsection{Previous Research Efforts}

Caron et al. formulated a novel theoretical approach to identify the movement of non-bulk materials with a combination of proximity algorithms and belief functions [8]. In this approach each material was assumed to be coupled with an identification device while a roving unit equipped with a positioning receiver would simultaneously collect its localization coordinates and the identification codes of the surrounding materials [8]. Based on the collected data, a proximity mechanism would estimate the material coordinates assuming an ideal square-pattern of reader communications. A joint mass function resulted from the intersection of timely sequenced squares of communication centered at the reader locations where a given component was detected. Thus, if a given component had been sitting in the same location, the reader communications would ideally never result in a null intersection. Indeed, the physical intersection of squares centered at reader locations should spell out the location of the component. If the component had been displaced to a different location, a high level of conflict would indicate that the component had been actually moved.

Even though the novel and impressive contribution of this theoretical study. The intersection of multiple consecutive squares would invariable result in null intersections. At the same time, these null intersection would result in a high level of conflict that would indicate the displacement of the resource to a new location - whether this is true or not. In reality, empirical evidence would need to be used to validate monitoring approaches.

\section{BELIEF FRAMEWORK}

An innovative framework approach to monitor construction resources was developed. For this framework, it is supposed that the localization of each construction resource is estimated from data collected 
with infrastructure-less type of technologies. Thus, it is also assumed that each site resource is equipped with an identification transmitter that has a known maximum radius $\mathrm{R}$ of communication. Then, a moving rover with adequate receivers would collect its own location and the position of the surrounding resources. With the collected data, the appropriate algorithms -such as those developed by [3], [9] - would estimate the localization coordinates $(\mathrm{X}, \mathrm{Y})$ of each resource in consecutive times $t_{-n}, \ldots, t_{-3}, t_{-2}, t_{-1}$, and $t$. In this framework, the focus is placed on the coordinated estimates of the site resources instead of the reader locations. Then, the intersection between two consecutive estimates at times $t_{-1}$ and $t$ based on the intersection of equivalent radius of communication $\mathrm{R}$ is defined by the mass function in Eq. 2 and the level of conflict between the two consecutive intersections in Eq. 3.

$$
\begin{gathered}
m_{i, t_{q}, t_{s}}=m_{i, t_{q}} \cap m_{i, t_{s}} \in[0,1] \forall i, t \\
\Phi_{i, t_{q}, t_{s}}=1-m_{i, t_{q}, t_{s}} \in[0,1]
\end{gathered}
$$

Now, assume that the level of conflict for two consecutive estimates at times $t_{1}$ and $t_{2}$ is to be assessed as shown in Figure 1. From Eq. 2, the intersection between both observations is 1 and the corresponding level of conflict is 0 . This is true regardless of the level of intersection between the circles of maximum communication R (equal to $r_{1}$ ) around the estimates. In this Figure, even though in (a) the two estimates are separated by a considerable distance (almost two times $\mathrm{R}$ ), the formulation above results in a null level of conflict (intersection equal to 1) between the two consecutive time positions. This null level of conflict is paradoxically the same for two consecutive and very similar location estimates (b) and hence denotes a lack of granularity in the results.

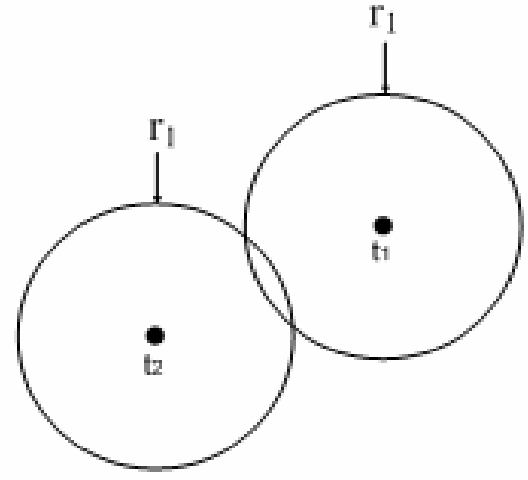

(a)

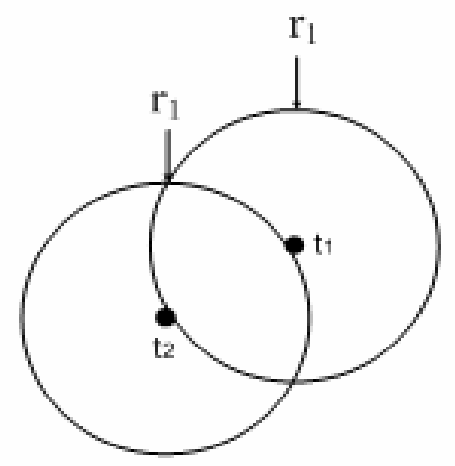

(b)

Fig. 1 Inability to properly monitor nearby resource locations with uniform mass probabilities

However, if additional inner circles of confidence are defined such as $r_{n}<\ldots r_{3}<r_{2}<r_{1}=R$, the granularity of the resulting level of conflict is increased. Take for instance the case were three $(n=3)$ confidence circles are defined around two consecutive localization estimates such as $r_{3}<r_{2}<r_{1}=R$ as shown in Figure 2 . Then, mass function values can be arbitrarily defined for each intercircle area -assume $\mathrm{m}\left(\mathrm{r}_{1}\right)=0.3, \mathrm{~m}\left(\mathrm{r}_{2}\right)=$ 0.5 , and $\mathrm{m}\left(\mathrm{r}_{3}\right)=0.2$. Now, while the level of conflict for case (b) is kept at a low value (0.09) and would not be indicative of displacement, the level of conflict for case (a) is 0.96 and suggests the displacement of the resource. 


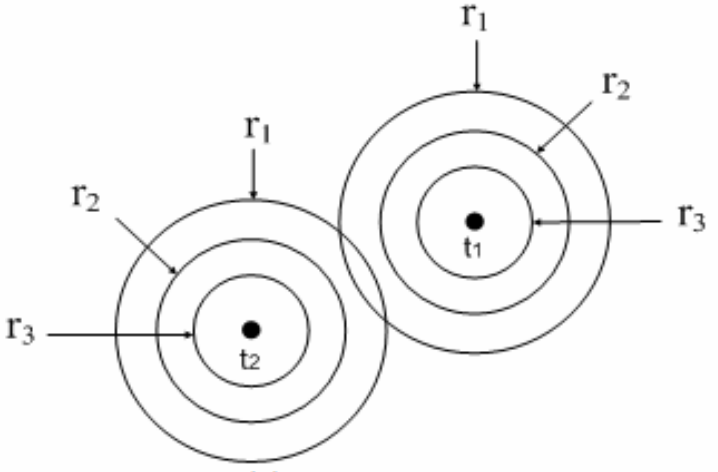

(a)

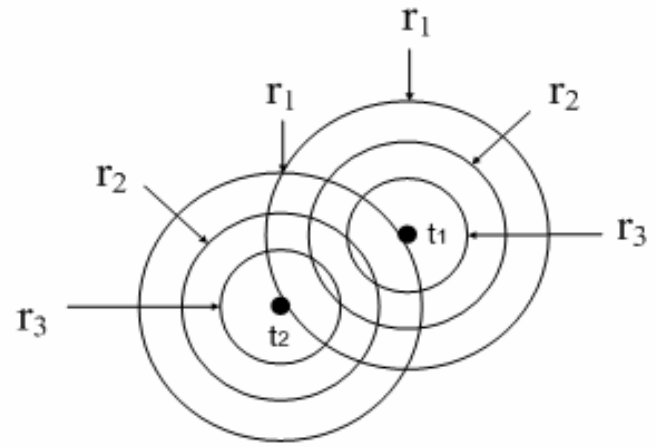

(b)

Fig. 2 Use of intercircle mass probabilities to feasibly monitor nearby resource locations

In reality, though, given the low accuracy and the anisotropy, harsh, and time-varying characteristics of construction scenarios, empirical observations denote that the distance between two localization estimates for a resource -the actual position of which has not varied - can eventually be larger than R. In this case, the level of conflict would be 1 and eventually lead to assume the change in the position of the resource and the generation of a false positive -the framework would imply the movement of a given resource when this has not occurred. Hence, the framework proposed in this study relies on the multiple comparison of individual pairs of time-sequential circles of confidence to determine whether a given resource is in a different position or not. For each pair, a level of conflict is defined. Thus, a resource is believed to actually be in a new set coordinates if and only if the sum of conflicting values as a result of intersecting pairs of confidence circles centered in the last and predecessor coordinated estimates exceeds a predefined threshold, as it is expressed in Eq. 4.

$$
\sum_{j=1}^{w} \Phi_{i, t_{n}, t_{n-j}} \geq \mathrm{C}, \quad \mathrm{C} \in[0, w[
$$

\section{EMPIRICAL VALIDATION}

A database containing localization records for a large quantity of material resources served for validation purposes. Since the localization of the material components was estimated with infrastructure-less technologies and recorded their actual movement, the displacement results generated by the framework were directly compared to the actual displacement of the components on the job site. Indeed, the framework successfully identified a minimum of $90 \%$ of the component displacements -and up to $100 \%$ for certain framework parameters. However, a small percentage (less than $4 \%$ ) of false positives was still generated. This small percentage of false positives was also minimized with the variation of framework parameters to less than $0.3 \%$ of the total number of data records. Overall, the validation results (see Table 1) assess the validity of the belief framework expressed by Eq. 4 .

Table 1 Results

\begin{tabular}{cccc}
\hline$w$ & C & $\begin{array}{c}\text { True } \\
\text { Positives }\end{array}$ & $\begin{array}{c}\text { False } \\
\text { Positives }\end{array}$ \\
\hline 1 & 0.182 & 29 & 48 \\
\hline 2 & 1.438 & 29 & 18 \\
\hline 3 & 2.075 & 31 & 11 \\
\hline 4 & 3.414 & 32 & 9 \\
\hline 5 & 4.009 & 31 & 6 \\
\hline 6 & 5.030 & 31 & 4 \\
\hline
\end{tabular}

\section{CONCLUSIONS AND RECOMMENDATIONS}

The unnecessary movement of site resources has negative effects on the cost, schedule, and safety project success indicators. To this date, there is no quantitative data to assess the magnitude of these unproductive movements. This study presents a novel 
approach to monitor the movement of site resources based on low-accuracy localization data. Building on top of the existing body of knowledge, this study have developed belief functions that can successfully track the movement of site resources in realistic construction sites characterized by harsh, time-varying, and anisotropic conditions. In reality, the validity of the framework has been extensively tested with data collected in real construction scenarios. To complete this study, the belief framework is being utilized to characterize the movement of resources on construction job sites and to evaluate the impact of these movements on construction productivity.

\section{ACKNOWLEDGEMENTS}

The author would like to thank to François Caron, Carl T. Haas, Emmanuel Duflos, Saiedeh Razavi, and Jongchul Song their support to the development of this study.

\section{REFERENCES}

[1] Caldas, C.H., Grau, D., and Haas, C.T. (2006). Using Global Positioning System to Improve Materials-Locating Processes on Industrial Projects. Journal of Construction Engineering and Management, 132(7), 741-749.

[2] Grau, D., Caldas, C. H., Goodrum, P. M., Haas, C. T., and Gong, J. (2009). "Benefits from Automating The Identification and Localization of Construction Components." Automation in Construction, 18(7), 903911.

[3] Grau, D., and Caldas, C.H. (2009). A Methodology for Automating the Identification and Localization of Construction Components on Industrial Projects. Journal of Computing in Civil Engineering, 23(1), 3-13. [4] Dempster, A.P. (1968). A generalization of Bayesian Inference. Journal of the Royal Statistical Society, Series B (30), 205-247.

[5] Shafer, G. (1976). A Mathematical Theory of Evidence. Princeton, Princeton University Press, New Jersey.
[6] Liu, L., and Yager, R. (2008). Classic Works of the Dempster-Shafer Theory of Belief Functions. In: Yager, R. and Liu, L., eds. Studies in Fuzziness and Soft Computing, vol. 219, Springer.

[7] Shafer, G. (1992). The Dempster-Shafer Theory. In: Shapiro, S.C. eds. Encyclopedia of Artificial Intelligence, Second Edition, Wiley, 330-331.

[8] Caron, F., Duflos, E., Haas, C., and Vanheeghe, P. (2007). Application du TBM pour la localization de noeuds de communication a partir de measures de proximite. Traitement du Signal, 2007, 24 (2), 153-164. [9] Song, J., Haas, C.T., and Caldas, C.H. (2006). Tracking the Location of Materials on Construction Job Sites. Journal of Construction Engineering and Management, 132(9), 911-918. 